\title{
Thermodynamic Ultrastability of a Polymer Glass Confined at the Micrometer Length Scale
}

\author{
Xavier Monnier* \\ Donostia International Physics Center, Paseo Manuel de Lardizabal 4, 20018 San Sebastián, Spain \\ Daniele Cangialosi ${ }^{\dagger}$ \\ Centro de Física de Materiales (CSIC-UPV/EHU), Paseo Manuel de Lardizabal 5, 20018 San Sebastián, Spain \\ and Donostia International Physics Center, Paseo Manuel de Lardizabal 4, 20018 San Sebastián, Spain
}

(Received 26 July 2018; published 25 September 2018)

\begin{abstract}
We employ fast scanning calorimetry to assess the thermodynamic state attained after a given cooling rate and the molecular mobility of glassy poly(4-tert-butylstyrene) confined at the micrometer length scale. We show that, for such a large confinement length scale, thermodynamic states with a fictive temperature $\left(T_{f}\right) 80 \mathrm{~K}$ below the polymer glass transition temperature $\left(T_{g}\right)$ are attained, which allows to bypass the geological timescales required for bulk glasses. Access to such states is promoted by a fast mechanism of equilibration. Importantly, the tremendous $T_{f}$ decrease takes place while the molecular mobility remains bulklike, indicating marked decoupling between vitrification kinetics and molecular mobility.
\end{abstract}

DOI: 10.1103/PhysRevLett.121.137801

Understanding vitrification in glass-forming systems still remains an unsolved problem in condensed matter physics [1]. The connection to the slowing down of molecular mobility is generally established. In particular, the conventional wisdom assumes that vitrification takes place when the timescale related to the molecular mobility, defined by a relaxation time, $\tau$, is of the order of the inverse of the cooling rate, $\beta_{c}[2,3]$. Below the glass transition temperature, $T_{g}$, the nonequilibrium glass spontaneously evolves toward equilibrium, a phenomenon known as structural recovery or physical aging [4,5]. Within the conventional wisdom on the glass transition, such recovery is exclusively related to $\tau$. Furthermore, a monotonic approach toward equilibrium is commonly encountered in glasses aged close to $T_{g}$ [4].

Given this premise, it is worth remarking that conceptually, vitrification and molecular mobility are different aspects of glassy dynamics [1,6]. The former entails information on how equilibrium is lost upon cooling the equilibrated melt or recovered in the glassy state. The thermodynamic state attained after vitrification or after aging in given conditions is commonly quantified using the concept of the fictive temperature, $T_{f}[7,8]$. This is defined as the temperature at which a glass in a certain thermodynamic state would be at equilibrium. In contrast, probing the molecular mobility requires the application of small perturbations - in particular, smaller than the amplitude of spontaneous fluctuations [9]. In this case, experiments or simulations are conducted at invariant $T_{f}$. Such conceptual difference is, with some exceptions [10], generally of no relevance in bulk glass formers [11-13], for which $T_{g}$ (or $T_{f}$ ) and the rate of physical aging are uniquely related to the molecular mobility.

Differently from the bulk case, experiments [14-19] and simulations $[20,21]$ on glasses geometrically confined at the nanoscale have revealed a decoupling between glass transition and physical aging on the one hand, and molecular mobility on the other, as discussed in several reviews [22-26]. In particular, this activity has shown that for polymeric glasses exhibiting a large amount of free interfacial area [27], negative $T_{g}$ deviations can be observed, even in the presence of essentially bulklike molecular mobility.

Another aspect of glassy dynamics challenging the conventional wisdom regards the existence of different equilibration mechanisms in the glassy state. In contrast to the standard behavior based on a single decay toward equilibrium for glasses aged close to $T_{g}$ [4] and after relatively small temperature jumps [28,29], recent experiments in a wide variety of glasses showed that two or more mechanisms of equilibrium recovery can be identified, provided that these experiments are performed substantially below $T_{g}$ and over timescales often exceeding several weeks [30-33]. The presence of different mechanisms of equilibration [34] is directly correlated to intermediate metastable states in the energy landscape. From the viewpoint of the timescales involved in glass equilibration, the slowest mechanism of equilibration exhibits the temperature dependence of the $\alpha$ process, generally associated with the glass transition, with large activation energy $[31,33]$. Conversely, the fast mechanisms generally display mild temperature dependence. 
In this Letter, we employ fast scanning calorimetry (FSC) to investigate the effect of size on the glass transition and molecular mobility of poly(4-tert-butylstyrene) (PtBS). This polymer has been previously shown to exhibit large confinement effects in terms of $T_{g}$ depression $[35,36]$. Here, we employ samples with a typical size in the micrometer scale. This is well beyond the length scale at which confinement effects on the glass transition are generally found [25,26,37]. The glass transition is characterized by assessing the limiting $T_{f}$ - that is, the one attained after cooling at a given rate. Furthermore, step response analysis [38], providing the complex specific heat, was employed to characterize the molecular mobility. Our results show tremendous $T_{f}$ negative deviations from bulk behavior. This can be as large as $80 \mathrm{~K}$ for the smallest sample. Furthermore, we find that such gross deviations are largely associated with a fast mechanism of equilibration. In contrast, molecular mobility exhibits bulklike behavior irrespective of the sample size.

P $t$ BS was purchased from Sigma Aldrich. It exhibits $M_{w}=130 \mathrm{~kg} / \mathrm{mol}$ and $M_{w} / M_{n}=2.7$, as determined by gel permeation chromatography. Samples were used as received. FSC experiments were performed using the Flash DSC 1 of Mettler Toledo, spanning a cooling/heating rate of $0.1-1000 \mathrm{~K} \mathrm{~s}^{-1}$ and reheating at $1000 \mathrm{~K} \mathrm{~s}^{-1}$ to assess the limiting $T_{f}$. In all cases, the latter was determined employing the Moynihan method [8] (see the Supplemental Material [39]). Four samples with masses 20, 50, 110, and $190 \mathrm{ng}$ were placed on the chips for FSC measurements. Sample geometries were characterized using confocal microscopy (see the Supplemental Material [39]). The so-obtained typical length scales of confinement, taken as the volume-to-area ratios $h=V / A$, were $2.5,4.0,5.2$, and $5.9 \mu \mathrm{m}$. Complementary measurements were carried out on bulk PtBS (mass $8 \mathrm{mg}$ ) by a DSC-Q2000 calorimeter from TA Instruments, calibrated with melting indium. The temperature control consisted of the DSC refrigerated cooling system by TA Instruments. DSC measurements were performed to obtain the cooling-rate-dependent limiting $T_{f}$ in the range $1-10 \mathrm{~K} \mathrm{~min}^{-1}$ and, by employing the modulated mode, the molecular mobility from the complex specific heat in a frequency range between $0.05-0.63 \mathrm{~Hz}$ (see the Supplemental Material [39]).

Step response analysis [38] consisted of applying a temperature jump of $2 \mathrm{~K}$ followed by isotherms of duration 0.1 and $0.5 \mathrm{~s}$. In such a way the instantaneous heat flow rate $H F(t)$ and the cooling rate $\beta_{c}(t)$ are obtained. These can be Fourier-transformed to determine the complex specific heat:

$$
C_{p}^{*}(\omega)=\frac{\int_{0}^{t_{p}} H F(t) e^{-i \omega t} d t}{\int_{0}^{t_{p}} \beta_{c}(t) e^{-i \omega t} d t} .
$$

From such transformation, the complex specific heat is obtained at the base frequency and higher harmonics:

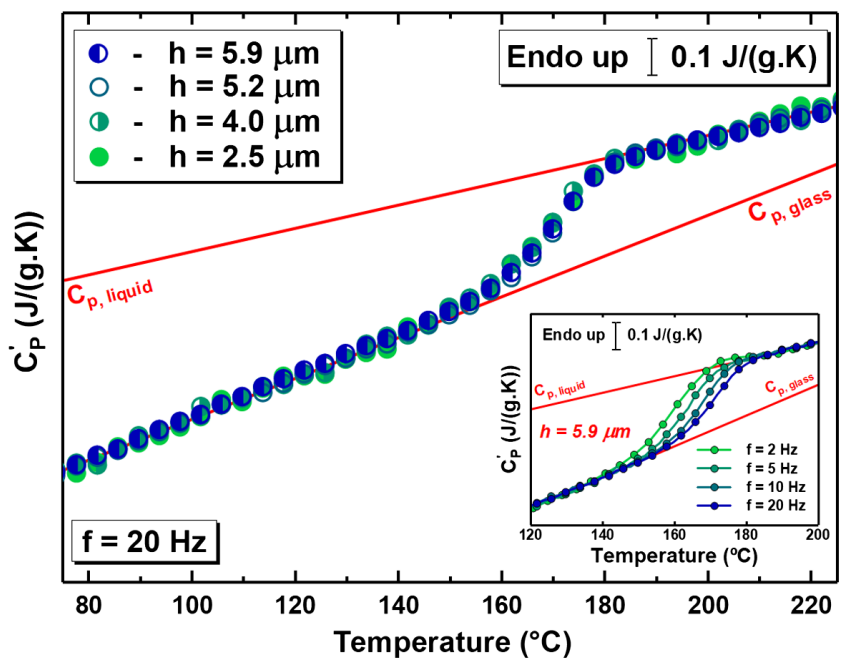

FIG. 1. Real part of the complex specific heat versus temperature obtained from step response analysis at $20 \mathrm{~Hz}$ by FSC for all investigated samples. The inset shows the real part of the complex specific heat versus temperature at different frequencies for the sample with $h=5.9 \mu \mathrm{m}$.

$\omega=k 2 \pi / t_{p}$, where $k$ is an integer. By changing $t_{p}$ and accessing higher harmonics, the explored frequency range was $2-140 \mathrm{~Hz}$. The $2 \mathrm{~K}$ temperature jump guarantees linearity of the measurement, as temperature fluctuations are generally larger than $2 \mathrm{~K}$ [42], and thereby, high-order contributions to $C_{p}^{*}$ are generally negligible. Hence, the intrinsic molecular mobility is accessed by this method.

Figure 1 depicts the real part of the complex specific heat $\left(C_{p}^{\prime}\right)$ versus temperature obtained from step response analysis by FSC at a frequency of $20 \mathrm{~Hz}$ for all investigated systems. As observed, all curves collapse on each other, indicating that at this frequency the molecular mobility is unaltered. The inset of Fig. 1 shows the frequencydependent $C_{p}^{\prime}$ for the sample with $h=5.9 \mu \mathrm{m}$ as a showcase. Increasing the frequency results in a shift toward higher temperatures at the $C_{p}^{\prime}$ step. A comprehensive overview of the size and frequency dependence on the relaxation time $\tau$, taken from the midpoint of the step in $C_{p}^{\prime}$, is reported on the left axis of Fig. 3. All data are superposed, indicating that the molecular mobility is unaltered. Furthermore, data on bulk $\mathrm{P} t \mathrm{BS}$ by conventional modulated DSC, though obtained at considerably lower frequencies, are in line with those of micrometric $\mathrm{P} t \mathrm{BS}$.

Figure 2 shows specific heat scans of all investigated systems obtained by FSC upon heating at $1000 \mathrm{~K} \mathrm{~s}^{-1}$ after cooling at the indicated rates. The common feature of all scans is the presence of a main endothermic peak in proximity to the glass transition and increasing in magnitude as the cooling rate is decreased. The location of such overshoot is essentially size independent, a result in line with reports showing no size dependence of the kink of the thickness [35] and the fluorescence intensity [36] in micrometric PtBS films. However, a difference in the main 

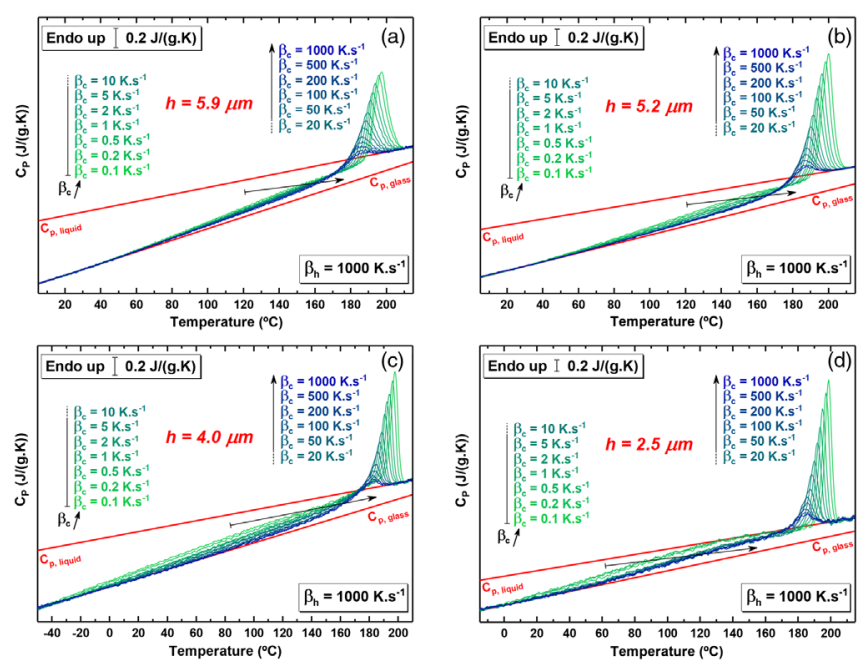

FIG. 2. Specific heat versus temperature upon heating at $1000 \mathrm{~K} \mathrm{~s}^{-1}$ after cooling at the indicated rates for the different length scales. For each sample, $C_{p, \text { glass }}$ and $C_{p \text {, liquid }}$ are taken from step response analysis at $10 \mathrm{~Hz}$ (see Fig. 1).

overshoot is evident, in that its intensity grows faster as the cooling rate is decreased in smaller samples. More importantly, apart from the overshoot in proximity to the glass transition, all samples exhibit pronounced excess $C_{p}$ with respect to the glass appearing at temperatures as small as $T_{g}-\sim 150 \mathrm{~K}$ for smaller samples. The latter was obtained from step response analysis considering the reversing specific heat: $C_{p(\mathrm{rev})}=\left(C_{p}^{\prime 2}+C_{p}^{\prime \prime 2}\right)^{0.5}$ (essentially coinciding with $C_{p}^{\prime}$; see Fig. 1 and Fig. S3 in the Supplemental Material [39]). The excess overshoot observed well below the glass transition exhibits the following main features: $(i)$ Its intensity increases when the cooling rate decreases, and it develops into a broad endothermic overshoot at the lowest rates. (ii) It is more pronounced and starts appearing at lower temperatures for smaller samples.

$C_{p}$ scans shown in Fig. 2 indicate that devitrification takes place in two steps, the first of which begins well below PtBS $T_{g}$. Furthermore, such early devitrification carries prominent excess specific heat, indicating the achievement of glasses with $T_{f}$ significantly below $\mathrm{P} t \mathrm{BS}$ $T_{g}$. An overview of the size and cooling-rate dependence of $T_{f}$ is presented in Fig. 3, on the right axis, for all systems including bulk $\mathrm{P} t \mathrm{BS}$ by standard DSC. Deviations from bulk behavior of the largest sample investigated by FSC $(h=5.9 \mu \mathrm{m})$ are almost negligible and hardly visible even at the lowest cooling rates. However, decreasing the sample size results in marked negative deviations from bulk behavior, most evident after cooling at low rates. These can be as large as $\sim 80 \mathrm{~K}$ for the sample with $h=2.5 \mu \mathrm{m}$ cooled at $q=0.1 \mathrm{~K} \mathrm{~s}^{-1}$. Furthermore, spanning the cooling rate by 4 orders of magnitude - that is, between 1000 and $0.1 \mathrm{~K} \mathrm{~s}^{-1}$ for the sample with $h=2.5 \mu \mathrm{m}$-involves the extraordinarily large $T_{f}$ variation of $80 \mathrm{~K}$. Figure 4

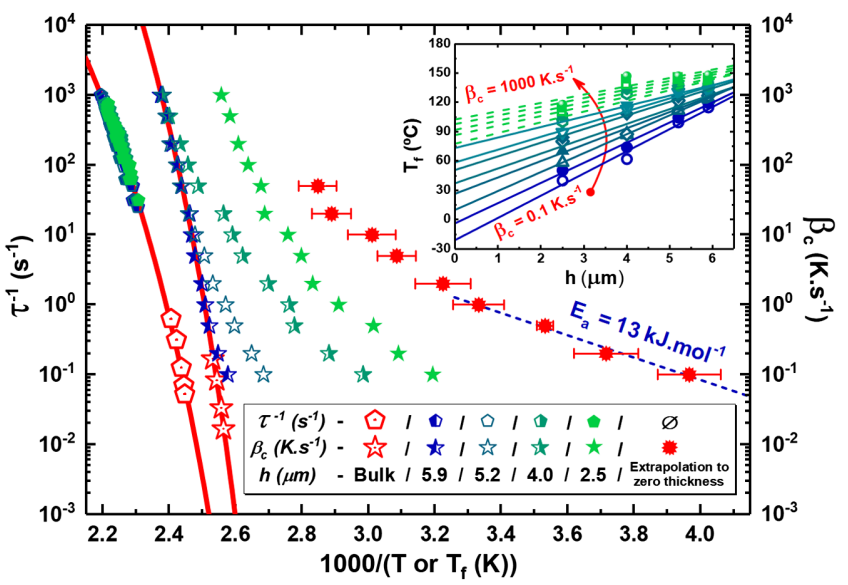

FIG. 3. Reciprocals of the relaxation time (left axis, pentagons) and cooling rate (right axis, stars) as functions of the inverse of temperature and fictive temperature, respectively. The solid lines are VFT fits with the parameters $B=2600 \mathrm{~K}, T_{0}=318 \mathrm{~K}$, and $A=11$ for fitting the relaxation time, and $A=14$ for the coolingrate-dependent $T_{f}$. The inset shows the confinement-length dependence of $T_{f}$ at different cooling rates. The dashed and solid lines are linear fits of the length-scale-dependent $T_{f}$ obtained at high and low cooling rates, respectively.

summarizes, in the enthalpy plot, the vastly different thermodynamic states attained by the smallest sample after cooling at 1000 and $0.1 \mathrm{~K} \mathrm{~s}^{-1}$. Furthermore, it is important to point out that in order to have a detectable $T_{f}$ decrease in bulk PtBS with the development of a broad endothermic overshoot well below the glass transition range, aging over several weeks at $313 \mathrm{~K}$ must be carried out (see Fig. S7 in

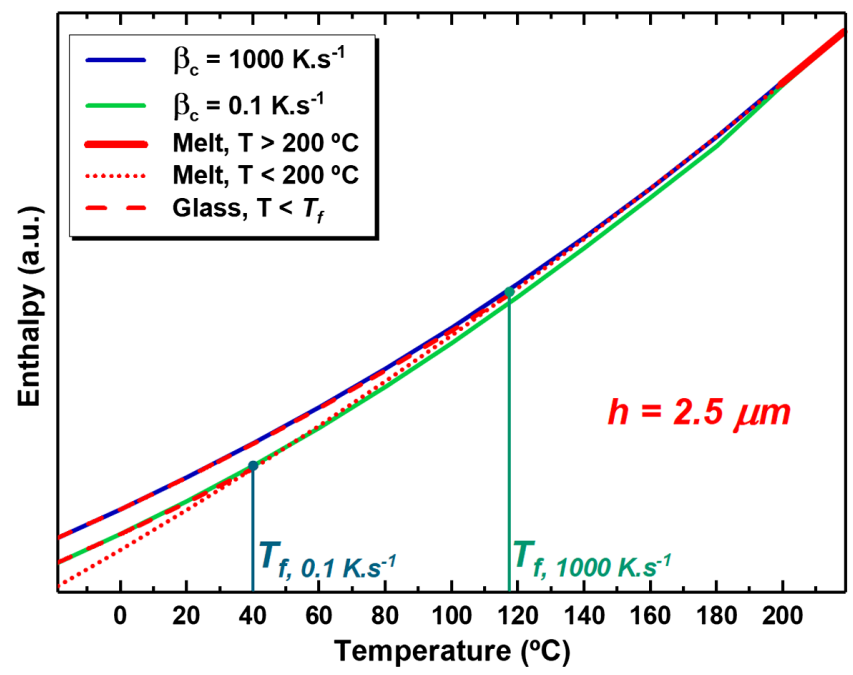

FIG. 4. Enthalpy as a function of temperature for a P $t$ BS sample with a characteristic length scale of $2.5 \mu \mathrm{m}$ on heating at $1000 \mathrm{~K} \mathrm{~s}^{-1}$ after cooling at $1000 \mathrm{~K} \mathrm{~s}^{-1}$ (blue line) and $0.1 \mathrm{~K} \mathrm{~s}^{-1}$ (green line). From the $C_{p \text {,liquid }}$ and $C_{p \text {,glass }}$ of Fig. 2(d), the melt enthalpy (red dotted line) and the glass enthalpy (red dashed lines) are shown. 
the Supplemental Material [39]). This covers a range of 6 orders of magnitude of timescales - that is, considerably larger than that relevant for cooling-rate-dependent determination of $T_{f}$ by FSC - and the observed $T_{f}$ reduction amounts to only $3 \mathrm{~K}$.

Altogether, our results highlight the following main points: (i) $\mathrm{P} t \mathrm{BS}$ samples with typical lengths in the micrometer range exhibit pronounced size dependence of the limiting $T_{f}$. (ii) Such dependence is unrelated to a modification of the molecular mobility, which instead is unaltered. (iii) The massive $T_{f}$ decrease observed in smaller samples, particularly at low cooling rates, is mostly associated with the presence of a broad endothermic overshoot located well below the PtBS glass transition.

The large deviation from the bulk glass transition at confinement lengths larger than the micrometer scale is, to our knowledge, the first report in this sense. Apart for some studies on polymer nanospheres showing $T_{g}$ deviations for submicrometer confinement length scales $[18,43]$, effects on the glass transition are generally observed below $100 \mathrm{~nm}$ [37]. Furthermore, it is important to point out that the observed size independence of $\tau$, cf. the left axis of Fig. 3, implies a marked decoupling between molecular mobility and glass transition in line with numerous previous observations [14-21]. Such decoupling is clearly evident for samples with $h=2.5$ and $4 \mu \mathrm{m}$, and to a minor extent for the sample with $h=5.2 \mu \mathrm{m}$. The largest sample investigated by FSC presents almost bulklike behavior. For such sample and bulk PtBS, the VFT law [44] $x=A+B /\left(T-T_{0}\right)$, where $x$ can be either $\log \beta_{c}^{-1}$ or $\log \tau$, can be applied. Identical values of $B$ and $T_{0}$ allow fitting $\tau$ and cooling-rate-dependent $T_{f}$ (see the caption of Fig. 3). This indicates that for these systems, complete coupling between molecular mobility and glass transition exists, as is generally observed in bulk glasses [11-13], in contrast to samples with smaller size.

An important aspect of our results is that the large $T_{f}$ decrease in microsized $\mathrm{P} t \mathrm{BS}$ originates from the presence of the low-temperature endothermic overshoot. This indicates the presence of a fast mechanism of equilibration, beyond the slow one with VFT behavior, already documented in previous studies [30-33]. Furthermore, the existence of a low-temperature overshoot appears to be rather ubiquitous in glasses of different nature, provided they are aged over prolonged times and far below $T_{g}$. This is the case of a liquid crystal [45], a metallic glass [46], and several high- $T_{g}$ polymers [47] aged at room temperature over several years. A low-temperature overshoot in the coefficient of thermal expansion was recently observed in polystyrene aged at room temperature over six months [48]. The presence of a prepeak or second glass transition in different glass formers-including water [49], polymers [50], and metallic glasses [51] — may be ascribed to a second mechanism of equilibration. While at present the molecular motion associated with such a fast mechanism needs to be identified, it is worth mentioning that the random first-order transition (RFOT) theory predicts a bimodal distribution of relaxation times [52]. The connection to volume-sensitive secondary relaxations $[53,54]$ may also be relevant, and further investigations in this sense should be warranted.

Within the context of the present study, it is noteworthy that the appearance of the low-temperature endothermic overshoot in micrometer-sized $\mathrm{P} t \mathrm{BS}$, providing $T_{f}$ decreases as large as $80 \mathrm{~K}$, is achieved exploring timescales ranging from milliseconds to seconds-that is, those relevant for FSC. Hence, a mild reduction of the length scale of confinement results in a tremendous reduction of the timescales of equilibration. Though on shorter confinement length scales, the presence of different mechanisms of equilibration has been recently documented. This is the case of freestanding PS films, which exhibit two kinks in their thickness when cooled at $0.5 \mathrm{~K} \mathrm{~min}^{-1}$ [55]. Stacked PS films aged far from $T_{g}$ for timescales not larger than several days were shown to exhibit two mechanisms of equilibrium recovery, the faster of them manifesting with a broad endothermic overshoot well below $T_{g}[56,57]$.

As a general rule, the results of the present study and those of other confined polymer glasses indicate that accessing thermodynamic states with low $T_{f}$ is strongly facilitated by the presence of a large amount of free interface [25-27]. The main consequence is that this kind of glass offers a formidable means to resolving challenges of the glass transition [58], thereby circumventing the geological timescales required to access low energies in bulk glasses [59] and offering alternative means to other routes such as physical vapor deposition [60]. The route based on enhancing the amount of free interface was recently exploited to show the existence of the ideal glass transition [61,62] in $30 \mathrm{~nm}$ stacked PS films [56].

Finally, insights on fundamental problems of the glass transition in confinement can be drawn from cooling-rateand size-dependent $T_{f}$ data. The inset of Fig. 3 shows the size dependence of $T_{f}$ obtained after different cooling rates. $T_{f}$ data related to high rates exhibit nonlinear size dependence. However, linear fitting of $T_{f}$ data obtained at low cooling rates can be pursued. Extrapolation of such linear fits to zero size delivers the cooling-rate-dependent $T_{f}$ shown in the main panel of Fig. 3. These data can be considered as representative of the free surface, and therefore the activation energy, $E_{a}$, that is associated with relaxation in such a region. An Arrhneius fit of data at the lowest cooling rates delivers $E_{a} \sim 13 \mathrm{~kJ} / \mathrm{mol}$. This low activation energy is in line with that reported in studies where the topmost free surface is probed at sufficiently low temperatures [63].

In conclusion, we have investigated the vitrification kinetics and the molecular mobility of $\mathrm{P} t \mathrm{BS}$ confined at the micrometer scale by FSC. The former was found to be greatly modified by confining this polymer at such a scale. 
In particular, we found large negative $T_{f}$ deviations from bulk behavior. These are largest for the smallest sample and reach $80 \mathrm{~K}$ at the lowest cooling rate. Importantly, $T_{f}$ deviations are decoupled from the molecular mobility, which is found to be bulklike and size independent. Our results show that the massive $T_{f}$ decrease arises from the presence of a weakly activated fast mechanism of equilibration, beyond the standard one with VFT temperature dependence. In summary, our work provides a route, based on increasing the amount of free interfacial area, to access low-energy states in glasses in short timescales. In such a way, information about so-far unexplored thermodynamic states of extraordinary importance for the comprehension of the glassy state can be achieved.

The authors thank the University of the Basque Country and the Basque Country government [Grant No. IT-654-13 $(\mathrm{GV})$ ], Departamento de Educación, Universidades e investigación, and the Spanish government [Grant No. MAT2015-63704-P, (MINECO/FEDER, UE)] for their financial support.

*xavier_monnier001@ehu.eus

†daniele.cangialosi@ehu.eus

[1] E. J. Donth, The Glass Transition (Springer-Verlag, Berlin Heidelberg, 2001).

[2] J. C. Mauro, P. K. Gupta, and R. J. Loucks, J. Chem. Phys. 126, 184511 (2007).

[3] J. W. Schmelzer, J. Chem. Phys. 136, 074512 (2012).

[4] A. J. Kovacs, Fortschr. Hochpolym. Forsch. 3, 394 (1963).

[5] L. C. E. Struik, Physical Aging in Amorphous Polymers and Other Materials (Technische Hogeschool Delft, Delft, Netherlands, 1977).

[6] G. Johari, Thermochim. Acta 523, 97 (2011).

[7] A. Tool, J. Am. Ceram. Soc. 29, 240 (1946).

[8] C. T. Moynihan, P. B. Macedo, C. J. Montrose, P. K. Gupta, M. A. De Bolt, J. F. Dill, B. E. Dom, P. W. Drake, A. J. Eastel, P. B. Elterman, R. P. Moeller, H. Sasabe, and J. A. Wilder, Ann. N.Y. Acad. Sci. 279, 15 (1976).

[9] H. Callen and R. Greene, Phys. Rev. 86, 702 (1952).

[10] C. G. Robertson, P. G. Santangelo, and C. M. Roland, J. Non-Cryst. Solids 275, 153 (2000).

[11] E. Donth, J. Korus, E. Hempel, and M. Beiner, Thermochim. Acta 304, 239 (1997).

[12] L.-M. Wang, V. Velikov, and C. A. Angell, J. Chem. Phys. 117, 10184 (2002).

[13] J. E. K. Schawe, J. Chem. Phys. 141, 184905 (2014).

[14] V. M. Boucher, D. Cangialosi, H. Yin, A. Schoenhals, A. Alegria, and J. Colmenero, Soft Matter 8, 5119 (2012).

[15] H. Yin, D. Cangialosi, and A. Schoenhals, Thermochim. Acta 566, 186 (2013).

[16] C. Zhang, V. M. Boucher, D. Cangialosi, and R. D. Priestley, Polymer 54, 230 (2013).

[17] V. Ramakrishnan, S. Harsiny, J. G. P. Goossens, T. L. Hoeks, and G. W. M. Peters, J. Polym. Sci. B 54, 2069 (2016).
[18] N. G. Perez-de Eulate, V. Di Lisio, and D. Cangialosi, ACS Macro Lett. 6, 859 (2017).

[19] S. Luo, Y. Chen, Y. Sha, G. Xue, E. Zhuravlev, C. Schick, X. Wang, D. Zhou, and L. Li, Polymer 134, 204 (2018).

[20] J. H. Mangalara, M. E. Mackura, M. D. Marvin, and D. S. Simmons, J. Chem. Phys. 146, 203316 (2017).

[21] W. Zhang, J. F. Douglas, and F. W. Starr, Proc. Natl. Acad. Sci. U.S.A. 115, 5641 (2018).

[22] Z. Yang, D. Peng, A. Clough, C.-H. Lam, and O. K. C. Tsui, Eur. Phys. J. Spec. Top. 189, 155 (2010).

[23] R. D. Priestley, D. Cangialosi, and S. Napolitano, J. NonCryst. Solids 407, 288 (2015).

[24] F. Kremer, M. Tress, and E. U. Mapesa, J. Non-Cryst. Solids 407, 277 (2015).

[25] D. Cangialosi, A. Alegria, and J. Colmenero, Prog. Polym. Sci. 5455, 128 (2016).

[26] S. Napolitano, E. Glynos, and N. B. Tito, Rep. Prog. Phys. 80, 036602 (2017).

[27] S. Napolitano, C. Rotella, and M. Wubbenhorst, ACS Macro Lett. 1, 1189 (2012).

[28] T. Hecksher, N. B. Olsen, K. Niss, and J. C. Dyre, J. Chem. Phys. 133, 174514 (2010).

[29] E. Lopez and S. L. Simon, Macromolecules 49, 2365 (2016).

[30] R. S. Miller and R. A. MacPhail, J. Chem. Phys. 106, 3393 (1997).

[31] D. Cangialosi, V. M. Boucher, A. Alegría, and J. Colmenero, Phys. Rev. Lett. 111, 095701 (2013).

[32] R. Golovchak, A. Kozdras, V. Balitska, and O. Shpotyuk, J Phys: Condens. Matter 24, 505106 (2012).

[33] I. Gallino, D. Cangialosi, Z. Evenson, L. Schmitt, S. Hechler, M. Stolpe, and B. Ruta, Acta Mater. 144, 400 (2018).

[34] P. Luo, P. Wen, H. Y. Bai, B. Ruta, and W. H. Wang, Phys. Rev. Lett. 118, 225901 (2017).

[35] N. G. Perez-de Eulate, M. Sferrazza, D. Cangialosi, and S. Napolitano, ACS Macro Lett. 6, 354 (2017).

[36] C. J. Ellison, M. K. Mundra, and J. M. Torkelson, Macromolecules 38, 1767 (2005).

[37] M. D. Ediger and J. A. Forrest, Macromolecules 47, 471 (2014).

[38] E. Shoifet, G. Schulz, and C. Schick, Thermochim. Acta 603, 227 (2015).

[39] See the Supplemental Material at http://link.aps.org/ supplemental/10.1103/PhysRevLett.121.137801 for further details on the characteristic confinement length scale, characterization of the molecular mobility, determination of the fictive temperature, standard differential scanning calorimetry experiments on bulk PtBS, reproducibility of experimental data, and thermal stability of samples, which includes Refs. [8,38,40,41].

[40] S. Weyer, M. Merzlyakov, and C. Schick, Thermochim. Acta 377, 85 (2001).

[41] X. Monnier, A. Saiter, and E. Dargent, Thermochim. Acta 658, 47 (2017).

[42] Y.Z. Chua, R. Zorn, O. Holderer, J. W. P. Schmelzer, C. Schick, and E. Donth, J. Chem. Phys. 146, 104501 (2017).

[43] C. Zhang, Y. Guo, and R. D. Priestley, Macromolecules 44, 4001 (2011). 
[44] P. G. Debenedetti and F. H. Stillinger, Nature (London) 410 , 259 (2001).

[45] J. Fan, E. I. Cooper, and C. A. Angell, J. Phys. Chem. 98, 9345 (1994).

[46] D. Aji and G. Johari, J. Chem. Phys. 142, 214501 (2015).

[47] N. G. Perez-De Eulate and D. Cangialosi, Phys. Chem. Chem. Phys. 20, 12356 (2018).

[48] L. Pradipkanti, M. Chowdhury, and D. K. Satapathy, Phys. Chem. Chem. Phys. 19, 29263 (2017).

[49] K. Amann-Winkel, C. Gainaru, P. H. Handle, M. Seidl, H. Nelson, R. Böhmer, and T. Loerting, Proc. Natl. Acad. Sci. U.S.A. 110, 17720 (2013).

[50] A. R. Berens and I. M. Hodge, Macromolecules 15, 756 (1982).

[51] D. V. Louzguine-Luzgin, I. Seki, T. Yamamoto, H. Kawaji, C. Suryanarayana, and A. Inoue, Phys. Rev. B 81, 144202 (2010).

[52] A. Wisitsorasak and P. G. Wolynes, J. Phys. Chem. B 118, 7835 (2014).

[53] J. C. Dyre and N. B. Olsen, Phys. Rev. Lett. 91, 155703 (2003).
[54] K. Ngai and M. Paluch, J. Chem. Phys. 120, 857 (2004).

[55] J. E. Pye and C. B. Roth, Phys. Rev. Lett. 107, 235701 (2011).

[56] V. M. Boucher, D. Cangialosi, A. Alegria, and J. Colmenero, Phys. Chem. Chem. Phys. 19, 961 (2017).

[57] V. M. Boucher, D. Cangialosi, A. Alegria, and J. Colmenero, J. Chem. Phys. 146, 203312 (2017).

[58] C. P. Royall, F. Turci, S. Tatsumi, J. Russo, and J. F. E. Robinson, J. Phys. Condens. Matter 30, 363001 (2018).

[59] J. Zhao, S. L. Simon, and G. B. McKenna, Nat. Commun. 4, 1783 (2013).

[60] S. F. Swallen, K. L. Kearns, M. K. Mapes, Y. S. Kim, R. J. McMahon, M. D. Ediger, T. Wu, L. Yu, and S. Satija, Science 315, 353 (2007).

[61] W. Kauzmann, Chem. Rev. 43, 219 (1948).

[62] J. H. Gibbs and E. A. DiMarzio, J. Chem. Phys. 28, 373 (1958).

[63] Z. Fakhraai and J. A. Forrest, Science 319, 600 (2008). 